\title{
BMJ Open Does undernutrition increase the risk of lost to follow-up in adults living with HIV in sub-Saharan Africa? Protocol for a systematic review and meta-analysis
}

Animut Alebel (10, , ${ }^{1,2}$ Daniel Demant, ${ }^{2,3}$ Pammla Petrucka, ${ }^{4,5}$ David Sibbritt ${ }^{2}$

To cite: Alebel A, Demant D, Petrucka $P$, et al. Does undernutrition increase the risk of lost to follow-up in adults living with HIV in subSaharan Africa? Protocol for a systematic review and meta-analysis. BMJ Open 2021;11:e048022. doi:10.1136/ bmjopen-2020-048022

- Prepublication history and additional supplemental material for this paper are available online. To view these files, please visit the journal online (http://dx.doi.org/10.1136/ bmjopen-2020-048022).

Received 16 December 2020 Accepted 16 November 2021

\section{Check for updates}

(c) Author(s) (or their employer(s)) 2021. Re-use permitted under CC BY-NC. No commercial re-use. See rights and permissions. Published by BMJ.

${ }^{1}$ College of Health Science, Debre Markos University, Debre Markos, Ethiopia

${ }^{2}$ School of Public Helath, Faculty of Health, University of Technology Sydney, Ultimo, New South Wales, Australia

${ }^{3}$ School of Public Health and Social Work, Faculty of Health, Queensland University of Technology, Kelvin Grove, QLD Australia

${ }^{4}$ University of Saskatchewan, Saskatoon, Saskatchewan, Canada

${ }^{5}$ Nelson Mandela African Institute of Science and Technology, Arusha, Tanzania

Correspondence to

Animut Alebel;

animut.a23@gmail.com

\section{ABSTRACT}

Introduction Undernutrition is considered a marker for poor prognosis among people living with HIV (PLHIV), particularly in sub-Saharan Africa (SSA), where undernutrition and HIV are both highly prevalent. Evidence suggests that undernutrition (body mass index $<18.5 \mathrm{~kg} /$ $\left.\mathrm{m}^{2}\right)$ is one of the main factors that significantly increases the risk of lost to follow-up (LTFU) in PLHIV. However, primary studies in SSA have reported inconsistent findings on the relationship between undernutrition and LTFU among adults living with HIV. To the best of our knowledge, no systematic review which aimed to summarise the available evidence. Hence, this review aims to determine the pooled effect of undernutrition on LTFU among adults living with HIV in SSA.

Methods and analysis PubMed, EMBASE, Web of Science, Scopus, and, for grey literature, Google Scholar will be systematically searched to include relevant articles published since 2005. Studies reporting the effect of undernutrition on LTFU in adults living with HIV in SSA will be included. The Newcastle-0ttawa Scale will be used for quality assessment. Data from eligible studies will be extracted using a standardised data extraction tool. Heterogeneity between included studies will be assessed using Cochrane $Q$-test and $\mathrm{I}^{2}$ statistics. The Egger's and Begg's tests at a $5 \%$ significance level will be used to evaluate publication bias. As heterogeneity is anticipated the pooled effect size will be estimated using a randomeffects model. The final effect size will be reported using the adjusted $\mathrm{HR}$ with a $95 \% \mathrm{Cl}$.

Ethics and dissemination Ethical approval is not required for a protocol for a systematic review. The results of this systematic review will be published in a peerreviewed journal and will be publicly available. PROSPERO registration number CRD42021277741.

\section{INTRODUCTION}

HIV remains a global public health challenge. Of the 38 million worldwide HIV cases at the end of 2019, more than two-thirds (25.7 million) were living in sub-Saharan Africa (SSA). ${ }^{1}$ Though there is no cure for HIV, antiretroviral (ARV) drugs can suppress viral replication and reduce or eliminate HIV transmission risk. ${ }^{2}$ ARV also assists people
Strengths and limitations of this study

To the best of our knowledge, this is the first systematic review and meta-analysis protocol designed to examine the effect of undernutrition on lost to follow-up among adults living with HIV in subSaharan Africa.

- The protocol included a comprehensive (detailed) searching strategy plans to include all eligible studies as much as possible.

- This protocol planned to quantify the final pooled effect size using adjusted HR to control for potential confounding factors.

- The protocol is reported according to the Preferred Reporting Items for Systematic Reviews and MetaAnalysis Protocol checklist to ensure quality.

- As different study designs and clinically heterogeneous participants are eligible for this protocol, these may be the possible sources of heterogeneity.

living with HIV (PLHIV) to live comparably healthy. ${ }^{3}$ The rapid scale-up of antiretroviral therapy (ART), especially in resource-limited settings, is one of the most remarkable achievements in the global efforts against HIV. ${ }^{4}$ Globally, access to ART has increased dramatically from $7 \%$ in 2005 to $67 \%$ in $2019 .^{15}$

ART is a daily medication, ${ }^{6} 7$ requiring a lifelong commitment to be effective. ${ }^{8}$ Lost to follow-up (LTFU) from ART profoundly affects success rates. LTFU is defined as when patients do not return to the ART clinic within 90 days (60 days after the next appointment) from the last clinic visit. ${ }^{9}$ It has become an emerging problem in many low- and middle-income countries (LMICs), including SSA. ${ }^{10}$ A meta-analysis from 42 LMICs found that nearly $35 \%$ of all patients initiated on ART either died or were LTFU at 36 months of follow-up. ${ }^{10}$ An additional metaanalysis from SSA has shown that up to $40 \%$ of patients were LTFU or died. ${ }^{12}$ PLHIV lost to ART are at higher risk of treatment failure, 
viral rebound, mortality and opportunistic infections (OIs) ${ }^{13} 14$ The common contributing factors for LTFU are low CD4, advanced WHO clinical staging (III and IV), poor ART adherence, low baseline body weight, weight loss $>10 \%$ and undernutrition (low body mass index (BMI)). ${ }^{15-23}$

Undernutrition is considered a marker for poor prognosis among PLHIV, particularly in SSA, where both undernutrition and HIV are highly prevalent. ${ }^{24}$ While both conditions are global problems, they are most prevalent in the world's poorest areas, such as SSA. SSA accounted for $23 \%$ of all people suffering from undernutrition and $68 \%$ of all PLHIV worldwide. ${ }^{25}{ }^{26}$ Undernutrition is characterised by a deficit in macronutrients and/or micronutrients, leading to body composition changes and diminished function. ${ }^{27} 28 \mathrm{HIV}$ and undernutrition are interrelated. HIV reduces food intake, reduces nutrient absorption and increases energy requirements. ${ }^{29-31}$ At the same time, undernutrition hastens disease progression and increases the occurrence and recurrence of OIs. ${ }^{30} 32$ Undernutrition significantly increases the risk of mortality, treatment failure, and LTFU among PLHIV. ${ }^{33-41}$

Studies have shown that undernutrition (ie, BMI $<18.5$ $\mathrm{kg} / \mathrm{m}^{2}$ ) is one of the main factors that significantly increases the risk of LTFU among adults living with HIV. ${ }^{42-47}$ This finding may reflect that undernourished patients are more likely to develop OIs and later died but were under-reported to the HIV clinics due to a passive reporting system. ${ }^{48}$ For example, a meta-analysis in SSA conducted by our team found that the risk of developing TB in undernourished adults living with HIV is twice that of well-nourished counterparts. ${ }^{49}$ In addition, undernourished patients may not be able to report to the health facility for ART refills and complete their appointments in the same manner as well-nourished patients. ${ }^{50}$ Additional study from Uganda has reported that overweight $\left(\right.$ BMI $>30 \mathrm{~kg} / \mathrm{m}^{2}$ ) patients living with HIV are at lower risk of LTFU compared with well-nourished patients living with HIV. ${ }^{48}$

There have been extensive primary studies on the relationship between undernutrition and LTFU among adults living with HIV in SSA. ${ }^{42} 45-48$ 50-57 However, these individual studies have reported inconsistent findings. Some studies showed that undernutrition significantly increases the risk of LTUF among adults living with HIV. ${ }^{42-47}$ Conversely, a few studies found that undernutrition and LTFU among adults living with HIV have no significant association. ${ }^{47}$ Estimating the pooled effect of undernutrition on LTFU among adults living with HIV is important to provide evidence for healthcare workers and policymakers in designing specific interventions to minimise undernutrition-related LTFU among adults living with HIV. However, to the best of our knowledge, there is no systematic review and meta-analysis, which summarised available evidence to show the pooled effect of undernutrition on LTFU among adults living with HIV in SSA. Thus, this review protocol has been designed to address this gap. This systematic review protocol is designed to estimate the pooled effect of undernutrition on LTFU among adults living with HIV in SSA. The authors will follow this protocol during the literature search, data analysis, and reporting of results.

\section{Review question}

Does undernutrition increase the risk of LTFU among adults living with HIV in SSA?

\section{The PICO framework}

- Participants/population: adults (aged $\geq 15$ years) living with HIV.

- Intervention(s)/exposure(s) group: undernourished adults living with HIV.

- Comparator(s)/control group: well-nourished adults living with HIV.

- Outcome(s) of interest: LTFU from ART.

\section{METHODS AND ANALYSIS}

\section{Information sources and search strategy}

This systematic review protocol is prepared following the Preferred Reporting Items for Systematic Reviews and Meta-Analysis Protocol (PRISMA) checklist (online supplemental additional file 1). ${ }^{58}$ The following databases will be searched: PubMed, EMBASE (Elsevier), Web of Science, Scopus, and, for grey literature, Google Scholar (see online supplemental additional file 2 for draft search strategy for PubMed). Additional studies will be identified through a review of reference lists of included studies. An updated search will be undertaken prior to the final manuscript submission in order to retain currency. Search limitation will include English publications, human subjects and a publication date since 2005 aligning with the first year in which payment-free ART was available in most African countries. A line-by-line search will be conducted from each database, and a separate searching strategy will be developed for each database, depending on the functions and search interface of databases. The search findings will be exported and managed through Covidence software.

\section{Study selection and eligibility criteria}

The systematic review will consider all studies reporting the association between undernutrition and LTFU among adults (aged $\geq 15$ years) living with HIV in SSA. Studies reported the association between undernutrition and LTFU in relative risk (RR) or HR will be considered for meta-analysis. Studies providing incomplete data, descriptive statistics only, review articles, case reports, editorial comments, non-aligned outcomes of interest, conference papers and qualitative studies will be excluded. Additionally, studies conducted among HIV-infected pregnant women will be excluded as the risk of undernutrition, and nutritional assessment tools used for pregnant women are distinct from non-pregnant individuals. ${ }^{59} \mathrm{Arti}-$ cles including only malnourished adults living with HIV will also not be considered for this review due to a lack of controls (ie, well-nourished adults living with HIV). 


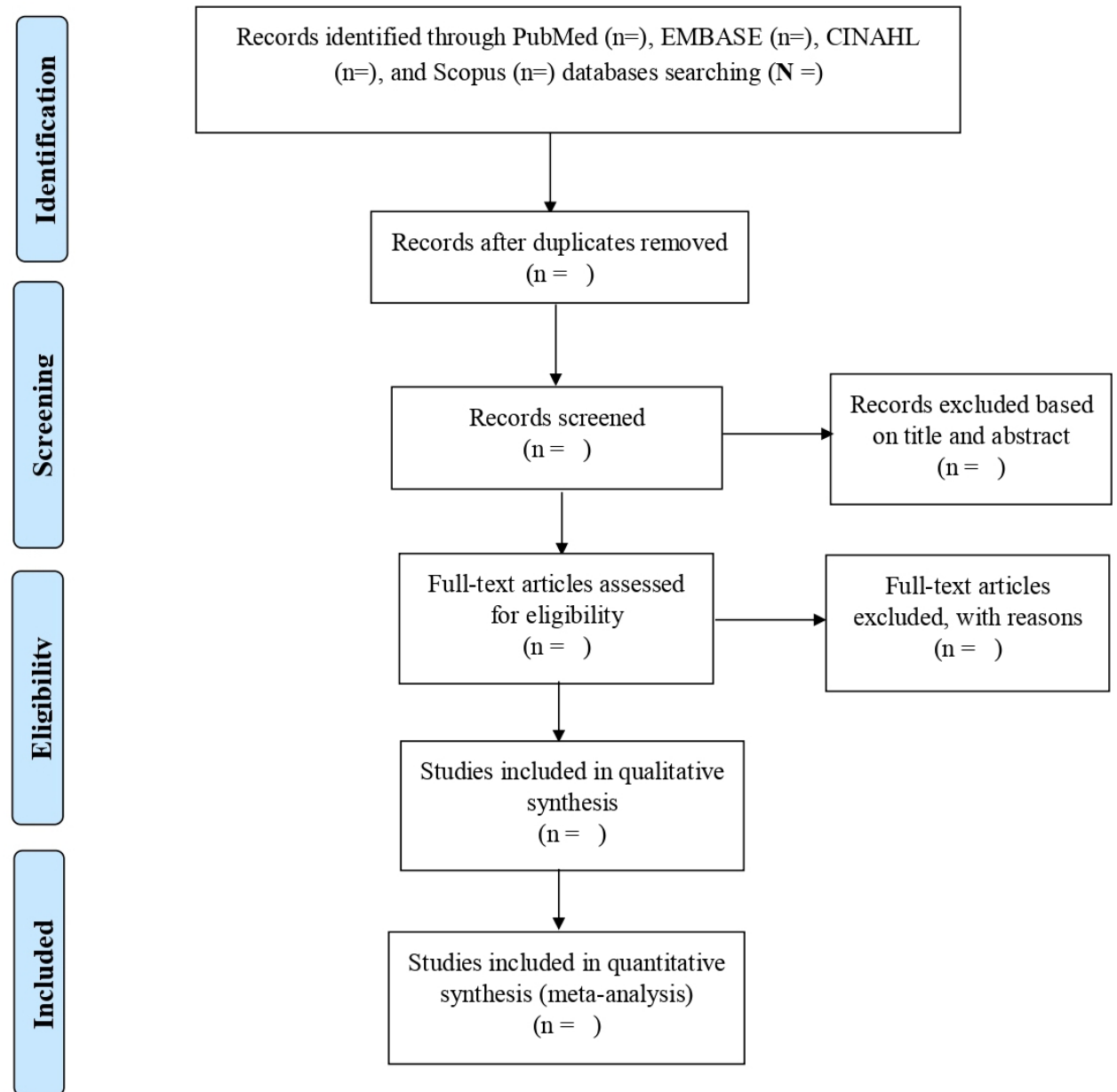

Figure 1 Flow chart of study selection for a systematic review and meta-analysis of the effect of undernutrition on LTFU in adults living with HIV in SSA. LTFU, lost to follow-up; SSA, sub-Saharan Africa.

The primary author (AA) will assess the titles and abstracts of potentially relevant studies. Then, two reviewers, (AA and $\mathrm{DD}$ ), will determine the inclusion and exclusion of full-texts based on the predetermined criteria. A third reviewer will be invited in cases of disagreement. The flow chart for this systematic review is described in figure 1.

\section{Data extraction and management}

A standardised data extraction tool will be adapted from the Joanna Briggs Institute (JBI) ${ }^{60}$ The following variables will be extracted: primary author name, publication year, study design, country/countries where the study was conducted, study/follow-up period, sample size, sex/gender of the participants, LTFU rate/proportion, adjusted confounders for LTFU (ie, sex, residence, age, distance from health facility, ART regimen, functional status, CD4 cell count, WHO clinical staging, ART adherence, OIs, cotrimoxazole preventive therapy, and isoniazid preventive therapy) and adjusted HR (AHR) for time-to-event analysis/adjusted $\mathrm{OR} /$ adjusted risk ratio (ARR) with $95 \%$ CIs. Any queries on primary article data collection or critical appraisal will lead to contact with corresponding authors. Failure to make the necessary connection will result in the article being excluded from our review. The primary author (AA) will extract data from included studies. To assure data quality, extracted data will be double-checked by another author (DD).

\section{Outcomes}

The outcome of this review is LTFU among adults living with HIV. LTFU will be identified as events in which patients not returning to the ART clinic within 90 days (60 days after the next appointment) from the last clinic visit. $^{9}$ Undernourished adults living with HIV will be considered as exposed the group to estimate the effect size of undernutrition on LTFU. Undernutrition (underweight) reflects an individual with a BMI of less than 18.5 $\mathrm{kg} / \mathrm{m}^{2}$. The severity of undernutrition is further classified as severe $\left(\mathrm{BMI}<16 \mathrm{~kg} / \mathrm{m}^{2}\right)$, moderate (BMI $16-16.99 \mathrm{~kg} /$ $\mathrm{m}^{2}$ ) or mild (BMI $\left.17-18.48 \mathrm{~kg} / \mathrm{m}^{2}\right)^{61}$

\section{Risk of bias in individual studies}

The Newcastle-Ottawa Scale (NOS) quality assessment tool will be used to assess the risk of bias in individual studies. ${ }^{62}$ The NOS is a validated tool with grading from zero to nine for case-control and cohort studies. ${ }^{63}$ The tool has three components: selection, comparability, and outcome/exposure. The selection part is scored from zero to four stars, and the comparability is scored 
from zero to two stars. The outcome/exposure is mainly related to the statistical analysis and cofounding handling mechanisms, which is scored from zero to three stars. Furthermore, to minimise the subjective interpretation of bias from scoring the NOS, three reviewers (AA, DD, and DS) will assess the quality of individual studies with consensus being achieved on all instances. Inter-rater reliability will be assessed using Cohen's kappa statistics. Finally, the quality score of each study will be calculated as the sum of scores.

\section{Data synthesis}

All statistical analyses will be done using Stata (V.16) statistical software. The effect of undernutrition on LTFU will be estimated using the AHR by considering undernutrition as exposure variable. RRs, rate ratios and incidence density ratios will be directly used as hazard ratios. Adult HIV patients with $\mathrm{BMI} \geq 18.5 \mathrm{~kg} / \mathrm{m}^{2}$ will be considered non-exposed (control group). In order to adjust for primary studies reporting AHRs considering undernourished adults living with HIV as a reference category, new AHRs with their 95\% CIs will be estimated by considering the reciprocal of the reported AHRs to ensure consistency and uniformity. ${ }^{64}$ If a study does not report RR/HR but reports the regression coefficient $(\beta)$, we will undertake conversion into RR/HR by exponentiation of the coefficient (ie, $R R=\exp (\beta)) .{ }^{65}$ The effect size (pooled AHR) will be estimated based on two nutritional status categories (undernutrition vs well nourished). If reported AHR is based on the severity level of undernutrition (mild, moderate and severe), categories will be considered in the subgroup analyses. Finally, those studies reporting nutritional status (BMI) as a continuous variable and cross-sectional studies reported ORs which are not eligible for meta-analysis will be addressed using a narrative synthesis approach.

Heterogeneity between included studies will be assessed using Cochrane Q-test and $\mathrm{I}^{2}$ statistics. The $\mathrm{I}^{2}$ value will be interpreted as: $0 \%$ to $40 \%$ (might not be important); $30 \%$ to $60 \%$ (may represent moderate heterogeneity; $50 \%-90 \%$ (may represent substantial heterogeneity); and $75 \%-100 \%$ (considerable heterogeneity) ${ }^{66}$ As heterogeneity is anticipated, the pooled effect size and 95\% CI will be estimated using a random-effects model with logit transformation and back transformation. Finally, all relevant findings will be presented using text, tables, and forest plots.

\section{Subgroup and sensitivity analyses}

When considerable heterogeneity $\left(\mathrm{I}^{2} \geq 75 \%\right)$ is detected, potential sources of heterogeneity will be investigated using subgroup and meta-regression analyses. If appropriate, sub-group analyses will be conducted using different variables based on country, design, degree of undernutrition, sample size, and publication year. Furthermore, sensitivity analysis will be done by sequential removal of individual studies from the analysis.

\section{Meta-bias}

If more than eight individual studies are included in a meta-analysis, funnel plots will be used to assess publication bias graphically. ${ }^{67}$ The Egger's and Begg's tests at a $5 \%$ significance level will be used to confirm publication bias. ${ }^{68}$ In the presence of significant publication bias, trim and fill analyses will be done, and adjusted effect sizes will be reported.

\section{Patient and public involvement statement}

No involvement of patients or the public occurred during design, conduct, reporting or dissemination plans in this research.

\section{DISCUSSION}

LTFU from ART became a significant public health problem as ART was rapidly scaled up. ${ }^{410} 11$ Evidence shows that undernutrition significantly increases the risk of LTUF among adults living with HIV. ${ }^{42-47}$ Therefore, understanding the impact of undernutrition on LTFU is essential in designing appropriate interventions. However, there is no systematic review and metaanalysis summarising available evidence about the pooled effect of undernutrition on LTFU among adults living with HIV in SSA. Thus, we propose this systematic review and meta-analysis protocol which is feasible, attainable, and timely. This review is the first systematic review examining the effect of undernutrition on LTFU among adults living with HIV in SSA to the best of our knowledge.

This review will synthesise all the available studies reporting the effect of undernutrition on LTFU among adults living with HIV in SSA. Findings will be presented at conferences in poster or oral presentations. In addition, the final manuscript will be published in a peer-reviewed journal for broader dissemination. Furthermore, the final manuscript will report any reason for significant changes to the protocol following publication.

This review has a number of strengths and limitations. The final manuscript of this review will be reported in line with the PRISMA guidelines. ${ }^{69}$ This review will pursue a comprehensive search strategy to include all eligible studies. Predefined eligibility criteria concerning population, exposure, control and outcomes will be applied. The final pooled effect size will be reported using AHR to control potential confounders. Despite these strengths, it is essential to acknowledge the possible anticipated limitations. By limiting our search to studies published in the English language, we are potentially missing a few important non-English studies. Varying definitions of LTFU and follow-up duration of the included studies may limit the comparability of data. All studies included in our systematic review might not be included in our meta-analysis as studies might report BMI in different categories. 


\section{ETHICS AND DISSEMINATION}

Ethical approval is not required for a protocol for a systematic review. The results of this systematic review will be published in a peer-reviewed journal and will be publicly available.

Contributors AA: conceived the review protocol, designed the study methodology, drafted and revised the protocol, and designed the statistical analyses plan. DD and DS: conceived the review protocol, reviewed and edited the protocol, and reviewed the statistical analyses plan. PMP: critically reviewed the protocol and made revisions. All authors read and approved the final manuscript.

Funding The authors have not declared a specific grant for this research from any funding agency in the public, commercial or not-for-profit sectors.

Competing interests None declared.

Patient consent for publication Not applicable.

Provenance and peer review Not commissioned; externally peer reviewed.

Supplemental material This content has been supplied by the author(s). It has not been vetted by BMJ Publishing Group Limited (BMJ) and may not have been peer-reviewed. Any opinions or recommendations discussed are solely those of the author(s) and are not endorsed by BMJ. BMJ disclaims all liability and responsibility arising from any reliance placed on the content. Where the content includes any translated material, BMJ does not warrant the accuracy and reliability of the translations (including but not limited to local regulations, clinical guidelines, terminology, drug names and drug dosages), and is not responsible for any error and/or omissions arising from translation and adaptation or otherwise.

Open access This is an open access article distributed in accordance with the Creative Commons Attribution Non Commercial (CC BY-NC 4.0) license, which permits others to distribute, remix, adapt, build upon this work non-commercially, and license their derivative works on different terms, provided the original work is properly cited, appropriate credit is given, any changes made indicated, and the use is non-commercial. See: http://creativecommons.org/licenses/by-nc/4.0/.

ORCID iD

Animut Alebel http://orcid.org/0000-0003-2822-2062

\section{REFERENCES}

1 World health organisation: HIV/AIDS key facts, 2020. Available: https://www.who.int/news-room/fact-sheets/detail/hiv-aids [Accessed 23 Oct 2020].

2 Günthard HF, Saag MS, Benson CA, et al. Antiretroviral drugs for treatment and prevention of HIV infection in adults: 2016 recommendations of the International antiviral Society-USA panel. JAMA 2016;316:191-210.

3 Mayer KH, Venkatesh KK. Antiretroviral therapy as HIV prevention: status and prospects. Am J Public Health 2010;100:1867-76.

4 Fauci AS, Eisinger RW. PEPFAR - 15 Years and Counting the Lives Saved. N Engl J Med 2018;378:314-6.

5 World Health Organisation:. Antiretroviral therapy (art) coverage among all age groups, 2019. Available: https://www.who.int/gho/hiv/ epidemic_response/ART_text/en/ [Accessed 30 Oct 2020].

6 Deeks SG, Lewin SR, Havlir DV. The end of AIDS: HIV infection as a chronic disease. Lancet 2013;382:1525-33.

7 Brinkhof MWG, Pujades-Rodriguez M, Egger M, . Mortality of patients lost to follow-up in antiretroviral treatment programmes in resource-limited settings: systematic review and meta-analysis. PLoS One 2009;4:e5790.

8 Hughes AJ, Mattson CL, Scheer S, et al. Discontinuation of antiretroviral therapy among adults receiving HIV care in the United States. J Acquir Immune Defic Syndr 2014;66:80-9.

9 World Health Organization. Operations manual for delivery of HIV prevention, care and treatment at primary health centres in highprevalence, resource-constrained settings: edition 1 for fieldtesting and country adaptation, 2008. Available: https://apps.who.int/iris/ bitstream/handle/10665/44015/9789241597432_eng.pdf [Accessed 09 Sep 2021].

10 Fox MP, Rosen S. Retention of adult patients on antiretroviral therapy in low- and middle-income countries: systematic review and metaanalysis 2008-2013. J Acquir Immune Defic Syndr 2015;69:98-108.

11 Levi J, Raymond A, Pozniak A, et al. Can the UNAIDS 90-90-90 target be achieved? A systematic analysis of national HIV treatment cascades. BMJ Glob Health 2016;1:e000010.
12 Rosen S, Fox MP, Gill CJ, . Patient retention in antiretroviral therapy programs in sub-Saharan Africa: a systematic review. PLoS Med 2007; 4:e298.

$13 \mathrm{KL}: \mathrm{S}$. The importance of treatment adherence in HIV. American Journal of Managed Care 2013;19:s231-7.

14 Luebbert J, Tweya H, Phiri S, et al. Virological failure and drug resistance in patients on antiretroviral therapy after treatment interruption in Lilongwe, Malawi. Clin Infect Dis 2012;55:441-8.

15 Hassan AS, Mwaringa SM, Ndirangu KK, et al. Incidence and predictors of attrition from antiretroviral care among adults in a rural HIV clinic in coastal Kenya: a retrospective cohort study. BMC Public Health 2015;15:478.

16 Mutasa-Apollo T, Ford N, Wiens M, et al. Effect of frequency of clinic visits and medication pick-up on antiretroviral treatment outcomes: a systematic literature review and meta-analysis. J Int AIDS Soc 2017;20:21647

17 Karcher $\mathrm{H}$, Omondi A, Odera J, et al. Risk factors for treatment denial and loss to follow-up in an antiretroviral treatment cohort in Kenya. Trop Med Int Health 2007;12:687-94.

18 Berheto TM, Haile DB, Mohammed S. Predictors of loss to follow-up in patients living with HIV/AIDS after initiation of antiretroviral therapy. $N$ Am J Med Sci 2014;6:453-9.

19 Megerso A, Garoma S, Eticha T, et al. Predictors of loss to followup in antiretroviral treatment for adult patients in the Oromia region, Ethiopia. Hiv Aids 2016;8:83-92.

20 Ochieng-Ooko V, Ochieng D, Sidle JE, et al. Influence of gender on loss to follow-up in a large HIV treatment programme in Western Kenya. Bull World Health Organ 2010;88:681-8.

21 Takarinda KC, Harries AD, Shiraishi RW, et al. Gender-Related differences in outcomes and attrition on antiretroviral treatment among an HIV-infected patient cohort in Zimbabwe: 2007-2010. Int J Infect Dis 2015;30:98-105.

22 Siril HN, Kaaya SF, Smith Fawzi MK, et al. Clinical outcomes and loss to follow-up among people living with HIV participating in the NAMWEZA intervention in Dar ES Salaam, Tanzania: a prospective cohort study. AIDS Res Ther 2017;14:18.

23 Seifu W, Ali W, Meresa B. Predictors of loss to follow up among adult clients attending antiretroviral treatment at Karamara General Hospital, Jigjiga town, eastern Ethiopia, 2015: a retrospective cohort study. BMC Infect Dis 2018;18:280.

24 Süttmann U, Ockenga J, Selberg $\mathrm{O}$, et al. Incidence and prognostic value of malnutrition and wasting in human immunodeficiency virusinfected outpatients. J Acquir Immune Defic Syndr Hum Retrovirol 1995:8:239-46.

25 UNAIDS: Global HIV \& AIDS statistics - 2019 fact sheet. Available: https://www.unaids.org/en/resources/fact-sheet [Accessed 03 Sep 2021].

26 Food and agriculture organization of the United nations: the state of food security and nutrition in the world, 2019. Available: http://www. fao.org/3/ca5162en/ca5162en.pdf[Accessed 03 Sep 2021].

27 Seres DS, . Surrogate nutrition markers, malnutrition, and adequacy of nutrition support. Nutr Clin Pract 2005;20:308-13.

28 Soeters PB, Reijven PL, van Bokhorst-de van der Schueren MA, et al. Van Gemert Wg: a rational approach to nutritional assessment. Clin Nutr 2008;27:706-16.

29 Kosmiski L, . Energy expenditure in HIV infection. Am J Clin Nutr 2011;94:1677S-82.

30 Duggal S, Chugh TD, Duggal AK. Hiv and malnutrition: effects on immune system. Clin Dev Immunol 2012;2012

31 Dikman AE, Schonfeld E, Srisarajivakul NC, et al. Human immunodeficiency virus-associated diarrhea: still an issue in the era of antiretroviral therapy. Dig Dis Sci 2015;60:2236-45.

32 de Pee S, Semba RD. Role of nutrition in HIV infection: review of evidence for more effective programming in resource-limited settings. Food Nutr Bull 2010;31:S313-44.

33 Ahoua L, Umutoni C, Huerga H, et al. Nutrition outcomes of HIV-infected malnourished adults treated with ready-to-use therapeutic food in subSaharan Africa: a longitudinal study. J Int AIDS Soc 2011;14:2.

34 Geng EH, Bwana MB, Muyindike W, et al. Failure to initiate antiretroviral therapy, loss to follow-up and mortality among HIVinfected patients during the pre-ART period in Uganda. $J$ Acquir Immune Defic Syndr 2013;63:e64-71.

35 Otwombe KN, Petzold M, Modisenyane T, et al. Factors associated with mortality in HIV-infected people in rural and urban South Africa. Glob Health Action 2014;7:25488.

36 Tchounga BK, Hønge BL, Eholie SP, et al. Effect of sex and age on outcomes among HIV-2-infected patients starting antiretroviral therapy in West Africa. AIDS 2016;30:2707-14.

37 Naidoo K, Yende-Zuma N, Augustine S, . A retrospective cohort study of body mass index and survival in HIV infected patients with and without TB co-infection. Infect Dis Poverty 2018;7:35. 
38 Tesfamariam K, Baraki N, Kedir H. Pre-ART nutritional status and its association with mortality in adult patients enrolled on art at Fiche hospital in North Shoa, Oromia region, Ethiopia: a retrospective cohort study. BMC Res Notes 2016:9:512.

39 Gupta A, Nadkarni G, Yang W-T, et al. Early mortality in adults initiating antiretroviral therapy (art) in low- and middle-income countries (LMIC): a systematic review and meta-analysis. PLoS One 2011;6:e28691

40 Ahmed M, Merga $\mathrm{H}$, Jarso $\mathrm{H}$, . Predictors of virological treatment failure among adult HIV patients on first-line antiretroviral therapy in Woldia and Dessie hospitals, northeast Ethiopia: a case-control study. BMC Infect Dis 2019;19:305.

41 Silverman RA, John-Stewart GC, Beck IA, et al. Predictors of mortality within the first year of initiating antiretroviral therapy in urban and rural Kenya: a prospective cohort study. PLoS One 2019;14:e0223411.

42 Bernard C, Balestre E, Coffie PA, et al. Aging with HIV: what effect on mortality and loss to follow-up in the course of antiretroviral therapy? the leDEA West Africa cohort collaboration. Hiv Aids 2018;10:239-52.

43 Kalinjuma AV, Glass TR, Weisser M, et al. Prospective assessment of loss to follow-up: incidence and associated factors in a cohort of HIV-positive adults in rural Tanzania. J Int AIDS Soc 2020;23:e25460.

44 Mekonnen N, Abdulkadir M, Shumetie E, et al. Yenit MK: incidence and predictors of loss to follow-up among HIV infected adults after initiation of first line anti-retroviral therapy at University of Gondar comprehensive specialized Hospital Northwest Ethiopia, 2018: retrospective follow up study. BMC Research Notes 2019;12:111

45 Teshome W, Belayneh M, Moges M, et al. Do loss to follow-up and death rates from art care vary across primary health care facilities and hospitals in South Ethiopia? A retrospective follow-up study. HIV AIDS 2015;7:167-74.

46 Hønge BL, Jespersen S, Nordentoft PB, et al. Loss to follow-up occurs at all stages in the diagnostic and follow-up period among HIV-infected patients in Guinea-Bissau: a 7-year retrospective cohort study. BMJ Open 2013;3:e003499.

47 Tweya H, Oboho IK, Gugsa ST, et al. Loss to follow-up before and after initiation of antiretroviral therapy in HIV facilities in Lilongwe, Malawi. PLoS One 2018;13:e0188488.

48 Kiwanuka J, Mukulu Waila J, Muhindo Kahungu M, et al. Determinants of loss to follow-up among HIV positive patients receiving antiretroviral therapy in a test and treat setting: a retrospective cohort study in Masaka, Uganda. PLoS One 2020;15:e0217606.

49 Alebel A, Demant D, Petrucka P, et al. Effects of undernutrition on mortality and morbidity among adults living with HIV in sub-Saharan Africa: a systematic review and meta-analysis. BMC Infect Dis 2021:21:1.

50 Opio D, Semitala FC, Kakeeto A, et al. Loss to follow-up and associated factors among adult people living with HIV at public health facilities in Wakiso district, Uganda: a retrospective cohort study. BMC Health Serv Res 2019;19:628.

51 Vinikoor MJ, Joseph J, Mwale J, et al. Age at antiretroviral therapy initiation predicts immune recovery, death, and loss to followup among HIV-infected adults in urban Zambia. AIDS Res Hum Retroviruses 2014:30:949-55.

52 Mekonnen N, Abdulkadir M, Shumetie E, et al. Incidence and predictors of loss to follow-up among HIV infected adults after initiation of first line anti-retroviral therapy at University of Gondar comprehensive specialized Hospital Northwest Ethiopia, 2018: retrospective follow up study. BMC Res Notes 2019;12:111.

53 Moyo F, Chasela C, Brennan AT, et al. Treatment outcomes of HIVpositive patients on first-line antiretroviral therapy in private versus public HIV clinics in Johannesburg, South Africa. Clin Epidemiol 2016;8:37-47.

54 Kalinjuma AV, Glass TR, Weisser M, et al. Prospective assessment of loss to follow-up: incidence and associated factors in a cohort of HIV-positive adults in rural Tanzania. J Int AIDS Soc 2020;23:e25460.

55 Teshale AB, Tsegaye AT, Wolde HF, . Incidence and predictors of loss to follow up among adult HIV patients on antiretroviral therapy in University of Gondar comprehensive specialized Hospital: a competing risk regression modeling. PLoS One 2020;15:e0227473.

56 Evans D, Maskew M, Sanne I, . Increased risk of mortality and loss to follow-up among HIV-positive patients with oropharyngeal candidiasis and malnutrition before antiretroviral therapy initiation: a retrospective analysis from a large urban cohort in Johannesburg, South Africa. Oral Surg Oral Med Oral Pathol Oral Radiol 2012;113:362-72.

57 Wekesa P, McLigeyo A, Owuor K, et al. Factors associated with 36-month loss to follow-up and mortality outcomes among HIV infected adults on antiretroviral therapy in central Kenya. BMC Public Health 2020;20:328.

58 Shamseer L, Moher D, Clarke M, et al. Preferred reporting items for systematic review and meta-analysis protocols (PRISMA-P) 2015 elaboration and explanation. BMJ 2015;350:97647.

59 Ververs M-T, Antierens A, Sackl A, et al. Which anthropometric indicators identify a pregnant woman as acutely malnourished and predict adverse birth outcomes in the humanitarian context? PLoS Curr 2013;5. doi:10.1371/currents.dis.54a8b618c1bc031ea140e3f2 934599c8. [Epub ahead of print: 07 Jun 2013].

60 Peters M, Godfrey-smith P, Mcinerney P. Guidance for the conduct of $\mathrm{JBI}$ scoping reviews Joanna Briggs Institute Reviewer's Manual South Australia. Joanna Briggs Institute, 2019.

61 Purnell JQ. Definitions, Classification, and Epidemiology of Obesity. In: Endotext [Internet. MDText. com, Inc., 2018

62 Peterson J, Welch V, Losos M, et al. The Newcastle-Ottawa scale (NOS) for assessing the quality of nonrandomised studies in metaanalyses. Ottawa: Ottawa Hospital Research Institute, 2011.

63 Luchini C, Stubbs B, Solmi M, et al. Assessing the quality of studies in meta-analyses: advantages and limitations of the Newcastle Ottawa scale. World J Metaanal 2017;5:80-4. doi:10.13105/wjma. v5.i4.80

64 Kleinbaum D, Klein M, E: P. Logistic regression a Self-Learning text. Berlin: Springer, 2010.

65 Wiest MM, Lee KJ, Carlin JB. Statistics for clinicians: an introduction to logistic regression. J Paediatr Child Health 2015;51:670-3.

66 Cumpston M, Li T, Page MJ, et al. Updated guidance for trusted systematic reviews: a new edition of the Cochrane Handbook for systematic reviews of interventions. Cochrane Database Syst Rev 2019;10:ED000142.

67 Sutton AJ, Abrams KR, Jones DR. Methods for meta-analysis in medical research. 348. Wiley Chichester, 2000.

68 Lin L, Chu H. Quantifying publication bias in meta-analysis. Biometrics 2018;74:785-94.

69 Liberati A, Altman DG, Tetzlaff J, et al. The PRISMA statement for reporting systematic reviews and meta-analyses of studies that evaluate health care interventions: explanation and elaboration. $J$ Clin Epidemiol 2009;62:e1-34. 